\title{
The correct derivation of the buckling equations of the shear-deformable FGM plates for the extended Kantorovich method
}

Ahmed Hassan Ahmed Hassan ( $15210457 @ s t u . o m u . e d u . t r)$

Ondokuz Mayıs Üniversitesi Mühendislik Fakültesi: Ondokuz Mayis Universitesi Muhendislik Fakultesi https://orcid.org/0000-0002-4880-0184

Naci Kurgan

Ondokuz Mayis University Faculty of Engineering: Ondokuz Mayis Universitesi Muhendislik Fakultesi

Nihat Can

Ondokuz Mayis University Faculty of Engineering: Ondokuz Mayis Universitesi Muhendislik Fakultesi

\section{Research Article}

Keywords: Extended Kantorovich method (EKM), Trefftz stability criterion, Functionally graded plates, Shear deformable plate theories, Plate buckling

Posted Date: April 28th, 2021

DOI: https://doi.org/10.21203/rs.3.rs-434537/v1

License: (9) This work is licensed under a Creative Commons Attribution 4.0 International License. Read Full License

Version of Record: A version of this preprint was published at Meccanica on January 18th, 2022. See the published version at https://doi.org/10.1007/s11012-021-01441-0. 


\title{
The correct derivation of the buckling equations of the shear-deformable FGM plates for the extended Kantorovich method
}

\author{
Ahmed Hassan Ahmed Hassan · Naci Kurgan · \\ Nihat Can
}

Received: date / Accepted: date

\begin{abstract}
This article presents the derivation of the elastic buckling equations and boundary conditions of shear-deformable plates in the frame of the extended Kantorovich method (EKM). Surveying the literature shows that those stability equations are often obtained using a wrong derivation by confusing them with the linear equilibrium condition. This work aims at providing the correct derivation that is built on the stability of the equilibrium condition. Buckling equations are derived for three different plate theories, namely, the first-order shear deformation plate theory (FSDT), the refined-FSDT, and the refined plate theory (RPT). This article is the first to implement the EKM based on a refined theory. Also, it is the first time to implement the refined-FSDT in buckling analysis. For the generic FGM plates, buckling equations derived based on the FSDT and refined-FSDT are both found to be simple and contain only the lateral displacements/rotations variations. On the other hand, those of the RPT, have coupled lateral and in-plane displacement variations, even if the physical neutral plate is taken as the reference plane. The considered plate is rectangular and under general inplane loads. The properties are of the plate continuously varying through its thickness which is assumed to change smoothly with a separable function in the two in-plane dimensions. The von Kármán nonlinearity is considered. The stability equations are derived according
\end{abstract}

A.H.A Hassan

Mechanical Engineering Department, Engineering Faculty, Ondokuz Mayis University, 55139 Atakum, Samsun, Turkey

Tel.: +90 (538) 8652306

Fax: +90 (362) 4576035

ORCiD: 0000-0002-4880-0184

E-mail: 15210457@stu.omu.edu.tr

N. Kurgan

Mechanical Engineering Department, Engineering Faculty, Ondokuz Mayis University, 55139 Atakum, Samsun, Turkey

ORCiD: 0000-0001-7297-7249

E-mail: naci.kurgan@omu.edu.tr

N. Can

Mechanical Engineering Department, Engineering Faculty, Ondokuz Mayis University, 55139 Atakum, Samsun, Turkey

ORCiD: 0000-0002-5741-0890

E-mail: 13210381@stu.omu.edu.tr 
to the Trefftz criterion, using the variational calculus. The solution methods of the obtained equations are out of the scope of this article.

Keywords Extended Kantorovich method (EKM) - Trefftz stability criterion · Functionally graded plates $\cdot$ Shear deformable plate theories $\cdot$ Plate buckling

\section{Introduction}

\subsection{Evolution of the EKM}

The extended Kantorovich method (EKM) had evolved from the so called classical Kantorovich method that was introduced in [II] as a solution method to the partial differential equations (PDE) by reducing the $d$-dimensional PDE that governs the problem into $d$ one dimensional (1D) ordinary differential equations (ODE). Obtaining those ODE's starts by approximating the $d$-dimensional solution function $\Psi\left(x_{1}, x_{2}, \ldots x_{d}\right)$ as the multiplication of $d$ uni-dimensional functions $\psi^{(k)}$, one in each dimension $x_{k}$, as shown in Eq. 四.

$$
\Psi\left(x_{1}, x_{2}, \ldots x_{d}\right) \approx \prod_{k=1}^{d} \psi^{(k)}
$$

There are two approaches to obtain the ODE's from the original PDE using the approximated solution function. Both start with the assumption that only one uni-directional function in the approximate solution is unknown, while all the others are given. The first approach is to substitute the approximate solution to the total potential energy integral, then use the variational calculus to derive the governing equation, which is now an ODE. In the second approach, as presented in [ [] , the approximate solution is substituted to the Galerkin form, i.e., the weak form, of the governing PDE and then the fundamental lemma of the variational calculus is implemented to obtain the ODE. Solving the obtained ODE terminates the steps of the classical Kantorovich method. The limitation of the classical Kantorovich method is that its accuracy depends on the initially guessed functions.

To overcome this limitation, [12] proposed the extended Kantorovich method (EKM), in which the obtained ODE's are solved iteratively until a satisfying convergence is achieved. In the EKM, the first guessed solution, i.e., the trail function, does not have to satisfy any of the boundary conditions. This is the main advantage of the EKM over the other variational methods like Rayleigh-Ritz and Galerkin's methods. EKM is often reported to provide accurate results with rapid convergence with minimum dependency on the trail function.

[36] further extended the EKM by introducing the use of multi-term trail functions, as shown in Eq. \, which describes the solution more accurately.

$$
\Psi\left(x_{1}, x_{2}, \ldots x_{d}\right) \approx \sum_{i=1}^{n} \prod_{k=1}^{d} \psi_{i}^{(k)}
$$

where $n$ is the number of terms. The multi-term EKM provided more accuracy and the ability to solve additional problems that were not solvable with the single-term EKM, e.g., the buckling of rectangular plate under pure in-plane shear loading, see [35,, 8$]$. By using $n$ terms in the approximate solution, the $d$-dimensional PDE is reduced to $d$ sets, each contains $n$ ODE's that have to be solved simultaneously. 
1.2 Applications of the EKM to the plate buckling problem

Among the first applications of the EKM were the plate bending problem [14] and the plate's eigenvalue problems, i.e., vibration [13] and buckling analyses [13, I, , 7 ]. Those researches had been followed by numerous ones in which the EKM was implemented in investigating the plate problems considering various complexities and configurations. A recent review of those articles is in [3I]]. It is observed that the vast majority of the researches that applied EKM on the buckling analysis of plates have considered the thin plates, and thus were based on the classical plate theory (CPT). Just a few are based on a shear deformation theory, see [25, 26, 27, 16]. Also, almost all the studies investigated the rectangular plates, except [34, 39] in which annular plates are investigated, and [8,9] in which skew plates are considered. The buckling of plates having a variable thickness is investigated using EKM in [4, 26]. Buckling of plates under linearly distributed in-plane forces is investigated using EKM in [27, 15, [17, Z], while the case of general in-plane loading is considered in [29]. It is also observed that all of the publications in this topic investigated the mechanical loading, except [9] which considered the buckling of thin plates under thermal loads. The effect of the elastic foundation on the buckling of plates is considered in [8,9,7]. The stability equations in [4, 34, 39, 33, 15, [77, [6] are obtained using Galerkin's method mentioned in Section [1], in which the approximate solution is substituted to the weak form of the two-dimensional stability equations. The other researches, i.e. $[9,4,25,26,27,29,28,30]$, implemented the energy approach of substituting the approximate solution to the integral of the total potential energy then deriving the stability equations using the variational calculus.

\subsection{Necessity of this derivation}

The important observation that necessitates the writing of this article is that except for the single article of [9], all the other publications those presented an energy approach based derivation of the buckling equations for EKM had wrongly confused the stability equations with the equilibrium equations. In all those publications, the stability equations had been derived from the state of stationary potential energy, i.e., the equilibrium condition that is shown in Eq. 3. They had derived the linear equilibrium equations and simply solve them as if they are eigenvalue stability equations. However, according to [10], this is fundamentally wrong.

$$
\delta \Gamma=0
$$

where $\Gamma$ is the potential energy; and $\delta$ is the variational operator. The terms "buckling equations" and "stability equations" are used interchangeably in this article. The main criteria on which the stability equations can be built are the neutral equilibrium method and the adjacent equilibrium method. The latter criterion is also known as the principle of minimum potential difference or the Trefftz criterion. According to the Trefftz criterion, stability equations are derived from the stationary state of the second variation of the potential energy at the equilibrium state prior to buckling, as shown in Eq. 团.

$$
\delta\left(\delta_{e}^{2} \Gamma\right)=0
$$

where $\delta_{e}^{2} \Gamma$ is the second variation of $\Gamma$ obtained at the equilibrium state prior to buckling, i.e., $\delta_{e}^{2} \Gamma=\left.\delta^{2} \Gamma\right|_{\delta \Gamma=0}$. Detailed discussion on the derivation the stability equation for plates can be found in [10]. An important note is that if wrong notations and interpretation are tolerated, then the wrongly derived stability equations in the literature resemble the correct 
stability equation. So, the solutions of those wrongly derived stability equations are not necessarily wrong.

This article aims at presenting the correct derivation of the static stability (buckling) equations of plates using the EKM. The derivation is provided in detail so fellow researchers can easily redo the derivation for other configurations of their concern. The problem configuration presented here is selected to be reasonably general, so many other interesting problems can be obtained by simplifying the results. The plate is selected to be of rectangular shape. The von Kármán geometrical nonlinearity is considered. The material of the plate is considered as functionally graded material (FGM), with arbitrary continuously changing properties.

Unlike the derivation presented in [9] that is based on CPT, the ones presented here are based on the shear-deformation plate theories. Three different theories are adopted. The first is the first-order shear deformation theory (FSDT). The FSDT is considered because, as stated in [22] and [21], there is no significant benefit of using higher-order shear deformation theories in the buckling analysis of plates. The second adopted theory is the refined-FSDT first proposed by [21], and recently modified by [18]. The third one is the refined plate theory (RPT), first proposed by [24], and numerous versions are presented ever since.

To the best of the authors' knowledge, this is the first article to implement EKM based on a refined plate theory. Also, it is the first time to implement the refined-FSDT in buckling analysis. The first derivation, which is based on FSDT, is presented in detail. While the second one is briefly presented due to the similarity in steps with the first one.

One last note to mention is that this article does not deal with the solution of the obtained ODE's. Solution of those equations is a separate topic. Surveying the literature shows that analytical, numerical and semi-analytical methods can all be used for that purpose.

\section{The derivation based on the FSDT}

The stability equations are derived here according to the Trefftz criterion, shown in Eq. 目. The reasonable steps are to first obtain the total potential energy $\Gamma$ of the plate. Then to find the its second variation $\delta^{2} \Gamma$, and impose the equilibrium conditions to the second variation, which gives $\delta_{e}^{2} \Gamma$. Next is to impose the concept of the Kantorovich method, which makes $\delta_{e}^{2} \Gamma$ a single-integral functional. The next step is to obtain the variation of $\delta_{e}^{2} \Gamma$, which is $\delta\left(\delta_{e}^{2} \Gamma\right)$. Last step is to equate $\delta\left(\delta_{e}^{2} \Gamma\right)$ to zero to obtain the stability equations and boundary conditions.

\subsection{Finding $\Gamma$}

$\Gamma$ of a rectangular plate having length of $2 a$, width of $2 b$ and thickness $h$, is given by

$$
\Gamma=U+V
$$

where $U$ is the strain energy, and $V$ is the potential energy of the applied loads. $U$ and $V$ are given as follows.

$$
\begin{gathered}
U=\frac{1}{2} \int_{-b}^{b} \int_{-a}^{a} \int_{-h / 2}^{h / 2}\left[\sigma_{x x} \varepsilon_{x x}+\sigma_{y y} \varepsilon_{y y}+\sigma_{z z} \varepsilon_{z z}+\tau_{x y} \gamma_{x y}+\tau_{x z} \gamma_{x z}+\tau_{y z} \gamma_{y z}\right] d z d x d y \\
V=\int_{-a}^{a}\left[\overline{N_{y y}} v_{(x, y)}+\overline{N_{x y}} u_{(x, y)}\right] d x+\int_{-b}^{b}\left[\overline{N_{x x}} u_{(x, y)}+\overline{N_{x y}} v_{(x, y)}\right] d y
\end{gathered}
$$


where $\sigma_{i i}$ and $\tau_{i j}$ are the normal and shear stresses, while $\varepsilon_{i i}$ and $\gamma_{i j}$ are the normal and shear strains, and $\overline{N_{i j}}$ are the in-plane applied loads at the reference plane, in the stated directions. In order to find those stress and strain components, the constitutive equations of FSDT are adopted.

$$
\bar{u}_{(x, y, z)}=u_{(x, y)}+\left(z-z_{0}\right) \theta_{(x, y)} \quad \bar{v}_{(x, y, z)}=v_{(x, y)}+\left(z-z_{0}\right) \varphi_{(x, y)} \quad \bar{w}_{(x, y, z)}=w_{(x, y)}
$$

where $\bar{u}, \bar{v}$, and $\bar{w}$ are the displacements of any point of the plate in the directions $x, y$, and $z$, respectively. $\theta$ and $\varphi$ are the rotations of a transverse normal about $y$-axis and $x$-axis, respectively. $z_{0}$ is the offset between the midplane and some reference plane through the thickness of the plate. $u, v$, and $w$ are the displacements of any point at the physical neutral plane. Considering the von Kármán nonlinearities, strains are related to the displacements by the following equations.

$$
\begin{array}{lll}
\varepsilon_{x x}=\bar{u}_{, x}+\frac{1}{2}\left(\bar{w}_{, x}\right)^{2} & \varepsilon_{y y}=\bar{v}_{, y}+\frac{1}{2}\left(\bar{w}_{, y}\right)^{2} & \varepsilon_{z z}=0 \\
\gamma_{x z}=\gamma_{z x}=\bar{u}_{, z}+\bar{w}_{, x} & \gamma_{y z}=\gamma_{z y}=\bar{v}_{, z}+\bar{w}_{, y} & \gamma_{x y}=\gamma_{y x}=\bar{u}_{, y}+\bar{v}_{, x}+\bar{w}_{, x} \bar{w}_{, y}
\end{array}
$$

Note that the subscripts after commas denote the partial derivatives in the stated directions. Stresses are related to the strains as follows.

$$
\begin{array}{ll}
\sigma_{x x}=\frac{E_{(z)}}{1-\mu^{2}}\left(\varepsilon_{x x}+\mu \varepsilon_{y y}\right) & \sigma_{y y}=\frac{E_{(z)}}{1-\mu^{2}}\left(\varepsilon_{y y}+\mu \varepsilon_{x x}\right) \\
\tau_{x y}=\tau_{y x}=\frac{E_{(z)}}{2(1+\mu)} \gamma_{x y} & \tau_{x z}=\tau_{z x}=\frac{k_{s} E_{(z)}}{2(1+\mu)} \gamma_{x z} \quad \tau_{y z}=\tau_{z y}=\frac{k_{s} E_{(z)}}{2(1+\mu)} \gamma_{y z}
\end{array}
$$

where $E_{(z)}$ is Young's elasticity modulus as a function of $z . \mu$ is the Poisson's ratio. $k_{s}$ is the shear correction factor that accommodates the discrepancy in the energy caused by the FSDT assumption of constant transverse shear stresses [22]. Substituting the constitutive

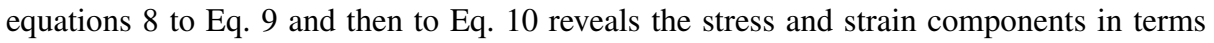
of $u, v, w, \theta, \varphi$, and their derivatives. Substituting those stresses and strains to Eq. $\square$ then integrating along $z$ gives $\Gamma$ as a double integral functional of $u_{(x, y)}, v_{(x, y)}, w_{(x, y)}, \theta_{(x, y)}$ and $\varphi_{(x, y)}$.

$$
\Gamma_{(u, v, w, \theta, \varphi)}=\int_{-b}^{b} \int_{-a}^{a}\left(\Gamma_{z}\right) d x d y+\int_{-a}^{a}\left[\overline{N_{y y}} v+\overline{N_{x y}} u\right] d x+\int_{-b}^{b}\left[\overline{N_{x x}} u+\overline{N_{x y}} v\right] d y
$$

where $\Gamma_{z}=$

$$
\begin{aligned}
& =\frac{A}{8}\left(\begin{array}{l}
2(1-\mu) K_{s}\left(\left(\theta+w_{, x}\right)^{2}+\left(\varphi+w_{, y}\right)^{2}\right)+4\left(u_{, x}{ }^{2}+v_{, y}{ }^{2}\right)+2(1-\mu)\left(u_{, y}+v_{, x}\right)^{2} \\
+\left(w_{, x}{ }^{2}+w_{, y}{ }^{2}\right)^{2}+4 w_{, x}{ }^{2}\left(u_{, x}+\mu v_{, y}\right)+8 \mu u_{, x} v_{, y}+4 w_{, y}{ }^{2}\left(\mu u_{, x}+v_{, y}\right) \\
+4(1-\mu) w_{, x} w_{, y}\left(u_{, y}+v_{, x}\right)
\end{array}\right) \\
& +\frac{B}{2}\left(\begin{array}{l}
(1-\mu)\left(\varphi_{, x}+\theta_{, y}\right)\left(u_{, y}+v_{, x}+w_{, x} w_{, y}\right)+\left(\mu \theta_{, x}+\varphi_{, y}\right)\left(2 v_{, y}+w_{, y}^{2}\right) \\
+\left(\theta_{, x}+\mu \varphi_{, y}\right)\left(2 u_{, x}+w_{, x}^{2}\right)
\end{array}\right) \\
& +\frac{D}{4}\left(4 \mu \theta_{, x} \varphi_{, y}+(1-\mu)\left(\theta_{, y}+\varphi_{, x}\right)^{2}+2\left(\theta_{, x}{ }^{2}+\varphi_{, y}{ }^{2}\right) \quad\right)
\end{aligned}
$$

where $A, B$, and $D$ are given as follows.

$$
A=\int_{-h / 2}^{h / 2} \frac{E_{(z)}}{1-\mu^{2}} d z \quad B=\int_{-h / 2}^{h / 2} \frac{\left(z-z_{0}\right) E_{(z)}}{1-\mu^{2}} d z \quad D=\int_{-h / 2}^{h / 2} \frac{\left(z-z_{0}\right)^{2} E_{(z)}}{1-\mu^{2}} d z
$$


$D$ and $A$ are the bending and extension stiffness. For the general case of variable thickness $h$ across the plate, $D$ and $A$ can be expressed as separable functions as shown below.

$$
D_{(x, y)}=D^{0} D^{x} D^{y} \quad A_{(x, y)}=A^{0} A^{x} A^{y}
$$

where $D^{0}$ and $A^{0}$ are constants; $D^{x}$ and $A^{x}$ are functions of $x ; D^{y}$ and $A^{y}$ are functions of $y$. If the thickness $h$ is constant, then $D^{x}=D^{y}=A^{x}=A^{y}=1$.

\subsection{Finding $\delta^{2} \Gamma$}

$\delta^{2} \Gamma$, the second variation of the total potential energy, is obtained as follows.

$$
\delta^{2} \Gamma=\int_{-b}^{b} \int_{-a}^{a}(\bar{\Gamma}) d x d y
$$

where $\bar{\Gamma}=$

$$
\begin{aligned}
& =\frac{A}{2}\left(\begin{array}{l}
(1-\mu) K_{s}\left(\left(\delta \theta+\delta w_{, x}\right)^{2}+\left(\delta \varphi+\delta w_{, y}\right)^{2}\right)+2\left(\left(\delta u_{, x}\right)^{2}+\left(\delta v_{, y}\right)^{2}\right) \\
+(1-\mu)\left(\delta u_{, y}+\delta v_{, x}\right)^{2}+4 \mu \delta u_{, x} \delta v_{, y}+2\left(\delta w_{, x}\right)^{2}\left(u_{, x}+\mu v_{, y}\right)+2\left(\delta w_{, y}\right)^{2}\left(\mu u_{, x}+v_{, y}\right) \\
+2(1-\mu) \delta w_{, x} \delta w_{, y}\left(u_{, y}+v_{, x}\right)+2(1-\mu)\left(\delta u_{, y}+\delta v_{, x}\right)\left(w_{, x} \delta w_{, y}+w_{, y} \delta w_{, x}\right) \\
+4 w_{, x} \delta w_{, x}\left(\delta u_{, x}+\mu \delta v_{, y}\right)+4 w_{, y} \delta w_{, y}\left(\mu \delta u_{, x}+\delta v_{, y}\right) \\
+4 w_{, x} w_{, y} \delta w_{, x} \delta w_{, y}+\left(w_{, y}\right)^{2}\left(3\left(\delta w_{, y}\right)^{2}+\left(\delta w_{, x}\right)^{2}\right)+\left(w_{, x}\right)^{2}\left(\left(\delta w_{, y}\right)^{2}+3\left(\delta w_{, x}\right)^{2}\right)
\end{array}\right) \\
& +B\left(\begin{array}{l}
(1-\mu)\left(\delta \varphi_{, x}+\delta \theta_{, y}\right)\left(\delta u_{, y}+\delta v_{, x}\right)+(1-\mu)\left(\delta \varphi_{, x}+\delta \theta_{, y}\right)\left(w_{, y} \delta w_{, x}+w_{, x} \delta w_{, y}\right) \\
+2\left(\mu \delta \theta_{, x}+\delta \varphi_{, y}\right)\left(\delta v_{, y}+w_{, y} \delta w_{, y}\right)+2\left(\delta \theta_{, x}+\mu \delta \varphi_{, y}\right)\left(\delta u_{, x}+w_{, x} \delta w_{, x}\right) \\
+\left(\delta w_{, x}\right)^{2}\left(\theta_{, x}+\mu \varphi_{, y}\right)+\left(\delta w_{, y}\right)^{2}\left(\mu \theta_{, x}+\varphi_{, y}\right)
\end{array}\right) \\
& +\frac{D}{2}\left(4 \mu \delta \theta_{, x} \delta \varphi_{, y}+(1-\mu)\left(\delta \theta_{, y}+\delta \varphi_{, x}\right)^{2}+2\left(\delta \theta_{, x}\right)^{2}+2\left(\delta \varphi_{, y}\right)^{2}\right)
\end{aligned}
$$

where $\delta u, \delta v, \delta w, \delta \theta, \delta \varphi, \delta u_{, x}, \delta u_{, y}, \delta v_{, x}, \delta v_{, y}, \delta w_{, x}$, and $\delta w_{, y}$ are the variational displacements and their derivatives. Note that all the terms of $V$ vanish when taking the second variation of the total potential energy. So, only $U$ contributes to $\delta^{2} \Gamma$.

\subsection{Finding $\delta_{e}^{2} \Gamma$}

The equilibrium of the flat plate implies that no lateral displacement or rotation occurs prior to the buckling point. Therefore, eliminating $w, \theta, \varphi$, and their derivatives from $\delta^{2} \Gamma$ in Eq. 14 gives $\delta_{e}^{2} \Gamma$, which is the second variation of the potential energy evaluated at the equilibrium state.

$$
\delta_{e}^{2} \Gamma=\int_{-b}^{b} \int_{-a}^{a}\left(\Gamma_{e}\right) d x d y
$$


where $\Gamma_{e}=$

$$
\begin{aligned}
& =\frac{A}{2}\left(\begin{array}{c}
(1-\mu) K_{s}\left(\left(\delta \theta+\delta w_{, x}\right)^{2}+\left(\delta \varphi+\delta w_{, y}\right)^{2}\right)+2\left(\left(\delta u_{, x}\right)^{2}+\left(\delta v_{, y}\right)^{2}\right) \\
+(1-\mu)\left(\delta u_{, y}+\delta v_{, x}\right)^{2}+4 \mu \delta u_{, x} \delta v_{, y}+2\left(\delta w_{, x}\right)^{2}\left(u_{, x}+\mu v_{, y}\right) \\
+2\left(\delta w_{, y}\right)^{2}\left(\mu u_{, x}+v_{, y}\right)+2(1-\mu) \delta w_{, x} \delta w_{, y}\left(u_{, y}+v_{, x}\right)
\end{array}\right) \\
& +B\left((1-\mu)\left(\delta \varphi_{, x}+\delta \theta_{, y}\right)\left(\delta u_{, y}+\delta v_{, x}\right)+2\left(\mu \delta \theta_{, x}+\delta \varphi_{, y}\right)\left(\delta v_{, y}\right)+2\left(\delta \theta_{, x}+\mu \delta \varphi_{, y}\right)\left(\delta u_{, x}\right)\right) \\
& +\frac{D}{2}\left(4 \mu \delta \theta_{, x} \delta \varphi_{, y}+(1-\mu)\left(\delta \theta_{, y}+\delta \varphi_{, x}\right)^{2}+2\left(\left(\delta \theta_{, x}\right)^{2}+\left(\delta \varphi_{, y}\right)^{2}\right)\right)
\end{aligned}
$$

In Eq. 15, the terms multiplied by the integral $B$ are the only ones that couple the variations of the lateral deflection and rotations $(\delta w, \delta \theta, \delta \varphi)$, with those of the in-plane displacements $(\delta u, \delta v)$. Equating the integral $B$ to zero eliminates that coupling. From Eq. ए2, $B$ is controlled by the material properties $E_{(z)}$ and $\mu$, and the the offset $z_{0}$ of the reference plane from the geometrical mid-plane through the thickness direction. The distance $z_{0}$ at which $B=0$ defines the neutral plane, at which no stresses or strains occur when the plate is under pure bending [38, 37]. Note that when $E_{(z)}$ is constant or symmetric about the midplane then $z_{0}$ has to vanish and the physical neutral plane coincides with the midplane.

Back to Eq. 대, the in-plane displacement terms $(u, v)$ multiplied by $\left(\delta w_{, x}\right)^{2},\left(\delta w_{, x}\right)^{2}$ and $\delta w_{, y} \delta w_{, x}$ present nothing but the integrals of the equilibrium state stress components $\sigma_{x x}, \sigma_{y y}$ and $\tau_{x y}$, respectively. Those integrals are called the force in-plane resultants $N_{x x}$, $N_{y y}$, and $N_{x y}$, and given by integrating the stress components of Eq. $\mathbb{}$ U along the thickness. Using the notation shown in Eq. 12, the force in-plane resultants are as follows.

$$
\left[\begin{array}{c}
N_{x x} \\
N_{y y} \\
N_{x y}
\end{array}\right]=\int_{-h / 2}^{h / 2}\left[\begin{array}{c}
\sigma_{x x} \\
\sigma_{y y} \\
\tau_{x y}
\end{array}\right] d z=\frac{A}{2}\left[\begin{array}{c}
2\left(u_{, x}+\mu v_{, y}\right) \\
2\left(\mu u_{, x}+v_{, y}\right) \\
(1-\mu)\left(u_{, y}+v_{, x}\right)
\end{array}\right]
$$

The resultant in-plane forces in the general case are variable through the plate's in-plane dimensions. They can be generally expressed in separated form as follows.

$$
N_{x x(x, y)}=N_{x x}^{0} N_{x x}^{x} N_{x x}^{y} \quad N_{y y(x, y)}=N_{y y}^{0} N_{y y}^{x} N_{y y}^{y} \quad N_{x y(x, y)}=N_{x y}^{0} N_{x y}^{x} N_{x y}^{y}
$$

For the case that the plate is under uniform or linearly varying in-plane loads acting in the principle directions $x$ and $y$, then the resultant in-plane forces will be equal to those applied forces [27,15]. Otherwise, if the applied in-plane loads have nonlinear distributions, then the resultant in-plane forces have to be obtained by solving the pre-buckling equilibrium problem [29]. The resultant forces do not have to be produced by direct mechanical load, as they may be created in plates under thermal loads, given that there is a restriction on expansion/retraction in one direction at least. For more about how to obtain the resultant thermal loads in plates see [5,9]. $\delta_{e}^{2} \Gamma$ can be rewritten as follows.

$$
\delta_{e}^{2} \Gamma=\int_{-b}^{b} \int_{-a}^{a}\left(\Gamma_{L}+\Gamma_{I}\right) d x d y
$$

where $\Gamma_{L}=$

$$
\begin{aligned}
& =\frac{A}{2}(1-\mu) K_{s}\left(\left(\delta \theta+\delta w_{, x}\right)^{2}+\left(\delta \varphi+\delta w_{, y}\right)^{2}\right) \\
& +\frac{D}{2}\left(4 \mu \delta \theta_{, x} \delta \varphi_{, y}+(1-\mu)\left(\delta \theta_{, y}+\delta \varphi_{, x}\right)^{2}+2\left(\left(\delta \theta_{, x}\right)^{2}+\left(\delta \varphi_{, y}\right)^{2}\right)\right) \\
& +\left(\left(\delta w_{, x}\right)^{2}\left(N_{x x}\right)+\left(\delta w_{, y}\right)^{2}\left(N_{y y}\right)+2 \delta w_{, x} \delta w_{, y}\left(N_{x y}\right)\right)
\end{aligned}
$$


and $\Gamma_{I}=$

$$
=\frac{A}{2}\left(2\left(\left(\delta u_{, x}\right)^{2}+\left(\delta v_{, y}\right)^{2}\right)+(1-\mu)\left(\delta u_{, y}+\delta v_{, x}\right)^{2}+4 \mu \delta u_{, x} \delta v_{, y}\right)
$$

In Eq. 188, the terms in $\delta_{e}^{2} \Gamma$ are rearranged in two groups. The first, $\Gamma_{L}$, contains the terms of variation of lateral displacement and rotation and no terms of variation of the in-plane displacements. While the second group, $\Gamma_{I}$, contains only the terms of variation of the in-plane displacement, and has no lateral displacement or rotation terms. In addition, $\delta_{e}^{2} \Gamma_{L}$ contains the force resultant components, while the $\delta_{e}^{2} \Gamma_{I}$ doesn't. Therefore, a further variation of $\delta_{e}^{2} \Gamma$ will result in an uncoupled set of equations. So, it is safe to only consider $\Gamma_{L}$ in the remaining steps of the derivation while being sure that this will not affect the resulting stability equations. The considered part in the remaining of the derivation is named $\delta_{e}^{2} \Gamma_{L}$.

\subsection{Imposing the concept of the Kantorovich method}

In this step, the unknown bivariate functions $\delta w_{(x, y)}, \delta \theta_{(x, y)}$, and $\delta \varphi_{(x, y)}$ are expressed as the summation of many terms, each is a multiplication of univariate functions of different variables, as illustrated in Eq. \. $\delta w_{(x, y)}, \delta \theta_{(x, y)}$, and $\delta \varphi_{(x, y)}$ are approximated as follows.

$$
\begin{aligned}
& \delta w_{(x, y)} \approx \sum_{i=1}^{n_{w}} f_{i(x)} F_{i(y)}=f F \\
& \delta \theta_{(x, y)} \approx \sum_{i=1}^{n_{\theta}} g_{i(x)} G_{i(y)}=g G \\
& \delta \varphi_{(x, y)} \approx \sum_{i=1}^{n_{\varphi}} m_{i(x)} M_{i(y)}=m M
\end{aligned}
$$

where $f, g$, and $m$ each is a single-row matrix contains the functions $f_{i}(x), g_{i}(x)$ and $m_{i}(x)$, respectively. $F, G$, and $M$ each is a single-column matrix contains the functions $F_{i}(y), G_{i}(y)$ and $M_{i}(y)$, respectively. Note that the function $\delta w_{(x, y)}$ is approximated using $n_{w}$ terms, while $n_{\theta}$ terms are used for $\delta \theta_{(x, y)}$, and $n_{\varphi}$ for $\delta \varphi_{(x, y)} . n_{w}, n_{\theta}$, and $n_{\varphi}$ are all independent of each other; i.e., each of the functions can be approximated using different number of terms. Substituting the approximations in Eq. [प] to $\Gamma_{L}$ in Eq. [8] gives

$$
\delta_{e}^{2} \Gamma_{L}=\int_{-b}^{b} \int_{-a}^{a}\left(\Gamma_{L}\right) d x d y
$$

where $\Gamma_{L}=$

$$
\begin{aligned}
& =\frac{A}{2}(1-\mu) K_{s}\left(\left(g G+f^{\prime} F\right)^{2}+\left(m M+f F^{\prime}\right)^{2}\right) \\
& +\frac{D}{2}\left(4 \mu\left(g^{\prime} G\right)\left(m M^{\prime}\right)+(1-\mu)\left(g G^{\prime}+m^{\prime} M\right)^{2}+2\left(\left(g^{\prime} G\right)^{2}+\left(m M^{\prime}\right)^{2}\right)\right) \\
& +\left(\left(f^{\prime} F\right)^{2}\left(N_{x x}\right)+\left(f F^{\prime}\right)^{2}\left(N_{y y}\right)+(1-\mu)\left(f^{\prime} F\right)\left(f F^{\prime}\right)\left(N_{x y}\right)\right)
\end{aligned}
$$


2.5 Stability equations and boundary conditions for $f_{(x)}, g_{(x)}$, and $m_{(x)}$

Assuming the functions of one direction are all known and differentiable, say $F, G$, and $M$, then the 2D functional $\delta_{e}^{2} \Gamma_{L}$ in Eq. 20 is reduced to a $1 \mathrm{D}$ functional of the functions $f, g$, and $m$. The stability equations are now obtainable by substituting $\delta_{e}^{2} \Gamma_{L}$ to the Trefftz criterion in Eq. 田.

$$
\begin{aligned}
& \delta\left(\delta_{e}^{2} \Gamma_{L}\right)=0= \\
& \int_{-a}^{a}\left(\delta_{f}\left[H_{1}+H_{y}+H_{x y 1}\right]+\delta_{g}\left[H_{2}\right]+\delta_{m}\left[H_{3}\right]+\delta_{f}{ }^{\prime}\left[H_{4}+H_{x}+H_{x y 2}\right]+\delta_{g}{ }^{\prime}\left[H_{5}\right]+\delta_{m}{ }^{\prime}\left[H_{6}\right]\right) d x
\end{aligned}
$$

where $H$ 's are as follows.

$$
\begin{aligned}
H_{1} & =A^{0} A^{x}(1-\mu) K_{s}\left[L_{\left(A^{y}, F^{\prime}, F^{\prime}\right)}^{y} f+L_{\left(A^{y}, F^{\prime}, M\right)}^{y} m\right] \\
H_{2} & =A^{0} A^{x}(1-\mu) K_{s}\left[L_{\left(A^{y}, G, G\right)}^{y} g+L_{\left(A^{y}, G, F\right)}^{y} f^{\prime}\right]+D^{0} D^{x}(1-\mu)\left[L_{\left(D^{y}, G^{\prime}, G^{\prime}\right)}^{y} g+L_{\left(D^{y}, G^{\prime}, M\right)}^{y} m^{\prime}\right] \\
H_{3} & =A^{0} A^{x}(1-\mu) K_{s}\left[L_{\left(A^{y}, M, F^{\prime}\right)}^{y} f+L_{\left(A^{y}, M, M\right)}^{y} m\right]+2 D^{0} D^{x}\left[\mu L_{\left(D^{y}, M^{\prime}, G\right)}^{y} g^{\prime}+L_{\left(D^{y}, M^{\prime}, M^{\prime}\right)}^{y} m\right] \\
H_{4} & =A^{0} A^{x}(1-\mu) K_{s}\left[L_{\left(A^{y}, F, G\right)}^{y} g+L_{\left(A^{y}, F, F\right)}^{y} f^{\prime}\right] \\
H_{5} & =2 D^{0} D^{x}\left[\mu L_{\left(D^{y}, G, M^{\prime}\right)}^{y} m+L_{\left(D^{y}, G, G\right)}^{y} g^{\prime}\right] \\
H_{6} & =D^{0} D^{x}(1-\mu)\left[L_{\left(D^{y}, M, G^{\prime}\right)}^{y} g+L_{\left(D^{y}, M, M\right)}^{y} m^{\prime}\right] \\
H_{x} & =2 N_{x x}^{0} N_{x x}^{x}\left[L_{\left(N_{x x}^{y}, F, F\right)}^{y} f^{\prime}\right] \\
H_{y} & =2 N_{y y}^{0} N_{y y}^{x}\left[L_{\left(N_{y y}^{y}, F^{\prime}, F^{\prime}\right)}^{y} f\right] \\
H_{x y 1} & =2 N_{x y}^{0} N_{x y}^{x}\left[L_{\left(N_{x y}^{y}, F^{\prime}, F\right)}^{y} f^{\prime}\right] \\
H_{x y 2} & =2 N_{x y}^{0} N_{x y}^{x}\left[L_{\left(N_{x y}^{y}, F, F^{\prime}\right)}^{y} f\right] \\
L^{y} \text { notation reads as } & L_{\left(\Psi_{0}, \Psi_{1}, \Psi_{2}\right)}^{y}=\int_{-b}^{b}\left[\Psi_{0}\left[\Psi_{1}\right]^{T}\left[\Psi_{2}\right]\right] d y
\end{aligned}
$$

Implementing the integration by parts on each integral in Eq. $2 \pi]$ that contains $\delta f^{\prime}, \delta g^{\prime}$, or $\delta m^{\prime}$, gives:

$$
\begin{aligned}
& \delta\left(\delta_{e}^{2} \Gamma_{L}\right)=0= \\
& \quad \int_{-a}^{a}\left(\delta_{f}\left[H_{1}+H_{y}+H_{x y 1}-H_{4}^{\prime}-H_{x}^{\prime}-H_{x y 2}^{\prime}\right]+\delta_{g}\left[H_{2}-H_{5}^{\prime}\right]+\delta_{m}\left[H_{3}-H_{6}^{\prime}\right]\right) d x \\
& \quad+\delta_{f}\left[H_{4}+H_{x}+H_{x y 2}\right]_{-a}^{+a}+\delta_{g}\left[H_{5}\right]_{-a}^{+a}+\delta_{m}\left[H_{6}\right]_{-a}^{+a}
\end{aligned}
$$

Since $\delta f, \delta g$, and $\delta m$ are all arbitrary, and to satisfy Eq. 23, all the following stability equations must hold.

$$
H_{1}+H_{y}+H_{x y 1}-H_{4}^{\prime}-H_{x}^{\prime}-H_{x y 2}^{\prime}=0 \quad H_{2}-H_{5}^{\prime}=0 \quad H_{3}-H_{6}^{\prime}=0
$$


In addition the following boundary conditions must be satisfied at $x \in\{-a,+a\}$.

\begin{tabular}{|c|c|c|c|}
\hline either & $f=0$ & or & $H_{4}+H_{x}+H_{x y 2}=0$ \\
\hline and either & $g=0$ & or & $H_{5}=0$ \\
\hline and either & $m=0$ & or & $H_{6}=0$ \\
\hline
\end{tabular}

When both the lateral displacement and rotations are restrained, the edge is said to be clamped $(\overline{\mathbf{C}})$. The simply supported edge $(\overline{\mathbf{S}})$ has the same restraints as the clamped one except that it can freely rotate about its axis. When there is no restriction on both the lateral displacement and rotations, the edge is called free $(\overline{\mathbf{F}})$. Those boundary conditions at the edges $x \in\{-a,+a\}$ are translated as follows.

$$
\begin{aligned}
& \overline{\mathbf{C}}: \quad f=0 \text { and } \quad g=0 \text { and } \quad m=0 \\
& \overline{\mathbf{S}}: \quad f=0 \text { and } g=0 \text { and } H_{6}=0 \\
& \overline{\mathbf{F}}: H_{4}+H_{x}+H_{x y 2}=0 \text { and } H_{5}=0 \text { and } H_{6}=0
\end{aligned}
$$

The buckling loads are obtained by solving the governing equations (24 and 25 ) as an eigenvalue problem for the critical resultant forces $N_{x x}, N_{y y}$, and/or $N_{x y}$.

This completes the derivation of the stability equations and boundary conditions for a given solution in the $x$ direction. The second set of governing equations and boundary conditions for a given solution in the $y$ direction can be obtained following the same steps.

2.6 Stability equations and boundary conditions for $F_{(y)}, G_{(y)}$, and $M_{(y)}$

To obtain the stability equations and boundary conditions for the other direction, the steps

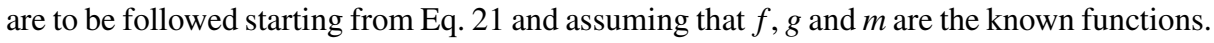

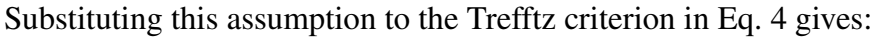

$$
\begin{aligned}
& \delta\left(\delta_{e}^{2} \Gamma_{L}\right)=0= \\
& \int_{-b}^{b}\left(\delta_{F}\left[T_{1}+T_{x}+T_{x y 1}\right]+\delta_{G}\left[T_{2}\right]+\delta_{M}\left[T_{3}\right]+\delta_{F}{ }^{\prime}\left[T_{4}+T_{y}+T_{x y 2}\right]+\delta_{G}{ }^{\prime}\left[T_{5}\right]+\delta_{M}{ }^{\prime}\left[T_{6}\right]\right) d y
\end{aligned}
$$


where $T$ 's are as follows.

$$
\begin{aligned}
T_{1} & =A^{0} A^{y}(1-\mu) K_{s}\left[L_{\left(A^{x}, f^{\prime}, f^{\prime}\right)}^{x} F+L_{\left(A^{x}, f^{\prime}, g\right)}^{x} G\right] \\
T_{2} & =A^{0} A^{y}(1-\mu) K_{s}\left[L_{\left(A^{x}, g, g\right)}^{x} G+L_{\left(A^{x}, g, f^{\prime}\right)}^{x} F\right]+2 D^{0} D^{y}\left[L_{\left(D^{x}, g^{\prime}, g^{\prime}\right)}^{x} G+\mu L_{\left(D^{x}, g^{\prime}, m\right)}^{x} M^{\prime}\right] \\
T_{3} & =A^{0} A^{y}(1-\mu) K_{s}\left[L_{\left(A^{x}, m, f\right)}^{x} F^{\prime}+L_{\left(A^{x}, m, m\right)}^{x} M\right]+D^{0} D^{y}(1-\mu)\left[L_{\left(D^{x}, m^{\prime}, g\right)}^{x} G^{\prime}+L_{\left(D^{x}, m^{\prime}, m^{\prime}\right)}^{x} M\right] \\
T_{4} & =A^{0} A^{y}(1-\mu) K_{s}\left[L_{\left(A^{x}, f, m\right)}^{x} M+L_{\left(A^{x}, f, f\right)}^{x} F^{\prime}\right] \\
T_{5} & =D^{0} D^{y}(1-\mu)\left[L_{\left(D^{x}, g, m^{\prime}\right)}^{x} M+L_{\left(D^{x}, g, g\right)}^{x} G^{\prime}\right] \\
T_{6} & =2 D^{0} D^{y}\left[\mu L_{\left(D^{x}, m, g^{\prime}\right)}^{x} G+L_{\left(D^{x}, m, m\right)}^{x} M^{\prime}\right] \\
T_{x} & =2 N_{x x}^{0} N_{x x}^{y}\left[L_{\left(N_{x x}^{x}, f^{\prime}, f^{\prime}\right)}^{x} F\right] \\
T_{y} & =2 N_{y y}^{0} N_{y y}^{y}\left[L_{\left(N_{y y}^{x}, f, f\right)}^{x} F^{\prime}\right] \\
T_{x y 1} & =2 N_{x y}^{0} N_{x y}^{y}\left[L_{\left(N_{x y}^{x}, f^{\prime}, f\right)}^{x} F^{\prime}\right] \\
T_{x y 2} & =2 N_{x y}^{0} N_{x y}^{y}\left[L_{\left(N_{x y}^{x}, f, f^{\prime}\right)}^{x} F^{x}\right] \\
L^{x} \text { notation reads as } & L_{\left(\Psi_{0}, \Psi_{1}, \Psi_{2}\right)}^{x}=\int_{-a}^{a}\left[\Psi_{0}\left[\Psi_{1}\right]^{T}\left[\Psi_{2}\right]\right] d x
\end{aligned}
$$

Implementing the integration by parts on each integral in Eq. 27 that contains $\delta F^{\prime}, \delta G^{\prime}$, or

\begin{tabular}{|c|c|c|c|c|c|}
\hline$\overline{\mathbf{C}}$ : & $F=0$ & and & $G=0$ & and & $M=0$ \\
\hline$\overline{\mathbf{S}}$ : & $F=0$ & and & $T_{5}=0$ & and & $M=0$ \\
\hline$\overline{\mathbf{F}}$ & $T_{4}+T_{y}+T_{x y 2}=0$ & and & $T_{5}=0$ & and & $T_{6}=0$ \\
\hline
\end{tabular}
$\delta M^{\prime}$, gives:

$$
\begin{aligned}
& \delta\left(\delta_{e}^{2} \Gamma_{L}\right)=0= \\
& \quad \int_{-b}^{b}\left(\delta_{F}\left[T_{1}+T_{x}+T_{x y 1}-T_{4}^{\prime}-T_{y}^{\prime}-T_{x y 2}^{\prime}\right]+\delta_{G}\left[T_{2}-T_{5}^{\prime}\right]+\delta_{M}\left[T_{3}-T_{6}^{\prime}\right]\right) d y \\
& \quad+\delta_{F}\left[T_{4}+T_{y}+T_{x y 2}\right]_{-b}^{+b}+\delta_{G}\left[T_{5}\right]_{-b}^{+b}+\delta_{M}\left[T_{6}\right]_{-b}^{+b}
\end{aligned}
$$

Since $\delta F, \delta G$, and $\delta M$ are all arbitrary, and to satisfy Eq. 29 , all the following stability equations must hold.

$$
T_{1}+T_{x}+T_{x y 1}-T_{4}^{\prime}-T_{y}^{\prime}-T_{x y 2}^{\prime}=0 \quad T_{2}-T_{5}^{\prime}=0 \quad T_{3}-T_{6}^{\prime}=0
$$

In addition the following boundary conditions must be satisfied at $y \in\{-b,+b\}$.

$$
\begin{array}{rllr}
\text { either } & F=0 & \text { or } & T_{4}+T_{y}+T_{x y 2}=0 \\
\text { and either } & G=0 & \text { or } & T_{5}=0 \\
\text { and either } & M=0 & \text { or } & T_{6}=0
\end{array}
$$

From Eq. B], the boundary conditions at the edges $y \in\{-b,+b\}$ can be written as follows 
Note that the obtained FSDT stability equations ( 24 and 30 ), and boundary conditions (25) and (31), resemble those found in the available literature, namely [25],26, 27]. The difference is that the ones presented here are correctly derived based on a stability criteria, while those found in [25, 26, 27] are wrongly obtained from the equilibrium state by vanishing the first variation of the potential energy.

Many other aspects can be easily included to the problem, for example, the effects of the elastic foundation, and the skew angle of the plate's geometry. Those aspects are not added to the derivation in this brief because they just add terms to the equations without contribution to the main aim of clarifying the derivation steps.

\section{The derivation based on the refined-FSDT}

The idea behind the refined-FSDT that first proposed by Qi [21], is to replace the shear correction factor $K_{s}$ with a suitable shear correction functions imposed to the transverse shear stress and strain in order to dictate their distributions through the thickness. Recently, Nguyen [18] merged that same concept with the one of decomposing the lateral displacement $w$ into bending and shear components $\left(w_{b}, w_{s}\right)$ to get rid of the dedicated rotation variables, $(\theta, \varphi)$. The constitutive equations of the refined-FSDT are as follows.

$$
\begin{aligned}
& \bar{u}_{(x, y, z)}=u_{(x, y)}-\left(z-z_{0}\right) \frac{\partial w_{b(x, y)}}{\partial x} \\
& \bar{v}_{(x, y, z)}=v_{(x, y)}-\left(z-z_{0}\right) \frac{\partial w_{b(x, y)}}{\partial y} \\
& \bar{w}_{(x, y, z)}=w_{s(x, y)}+w_{b(x, y)}
\end{aligned}
$$

As been mentioned above, the transverse shear strains are corrected by the function $\eta_{(z)}$ which gets transferred to the transverse shear stresses through the kinematic relations, and no additional correction factor $K_{s}$ is required. Thus transverse shear stresses and strains are given as follows.

$$
\begin{array}{ll}
\gamma_{x z}=\gamma_{z x}=\eta_{(z)}\left(\bar{u}_{, z}+\bar{w}_{, x}\right) & \tau_{x z}=\tau_{z x}=\frac{E_{(z)}}{2(1+\mu)} \gamma_{x z} \\
\gamma_{y z}=\gamma_{z y}=\eta_{(z)}\left(\bar{v}_{, z}+\bar{w}_{, y}\right) & \tau_{y z}=\tau_{z y}=\frac{E_{(z)}}{2(1+\mu)} \gamma_{y z}
\end{array}
$$

Note that the refined-FSDT herein refers to the general form of the theory, so, $\eta_{(z)}$ used here is general and does not have to be as the provided one in [18].

Due to the similarity of the steps, and to keep the brevity, the detailed steps of the derivation are omitted, and only a brief description is provided. Starting from the constitutive equations 33 considering the new formulae of the transverse shear stresses and strains in Eq. B4, and by implementing the same steps in Section $\square$ of finding the total potential energy $\Gamma$, then its second variation $\delta^{2} \Gamma$, then imposing the pre-buckling equilibrium state of zero lateral deflection $\left(w_{b}=w_{s}=0\right)$ to obtain $\delta_{e}^{2} \Gamma$ and then approximating $\left(\delta w_{b}, \delta w_{s}\right)$ as

$$
\delta w_{b(x, y)} \approx \sum_{i=1}^{n_{b}} f_{i(x)} F_{i(y)}=f F \quad \delta w_{s(x, y)} \approx \sum_{i=1}^{n_{s}} r_{i(x)} R_{i(y)}=r R
$$


then implementing the integration by parts, given that $F$ and $R$ are known, produce the following buckling equations.

$$
J_{1}+J_{y}+J_{x y 1}-J_{2}^{\prime}-J_{x}^{\prime}-J_{x y 2}^{\prime}+J_{3}^{\prime \prime}=0 \quad k_{1}+k_{y}+k_{x y 1}-k_{2}^{\prime}-k_{x}^{\prime}-k_{x y 2}^{\prime}=0
$$

The associated boundary conditions that must be satisfied at $x \in\{-a,+a\}$ are given below.

$$
\begin{aligned}
& \overline{\mathbf{C}}: \quad f=0 \text { and } \quad r=0 \text { and } f^{\prime}=0 \\
& \overline{\mathbf{S}} \text { : } \quad f=0 \text { and } \quad r=0 \text { and } J_{3}=0 \\
& \overline{\mathbf{F}}: \quad J_{2}+J_{x}+J_{x y 2}-J_{3}^{\prime}=0 \quad \text { and } \quad k_{2}+k_{x}+k_{x y 2}=0 \quad \text { and } \quad J_{3}=0
\end{aligned}
$$

where $J$ 's and $k$ 's are as follows.

$$
\begin{aligned}
& J_{1}=2 D^{0} D^{x}\left[L_{\left(D^{y}, F^{\prime \prime}, F^{\prime \prime}\right)}^{y} f+L_{\left(D^{y}, F^{\prime \prime}, F\right)}^{y} f^{\prime \prime}\right] \\
& J_{2}=4 D^{0} D^{x}(1-\mu)\left[L_{\left(D^{y}, F^{\prime}, F^{\prime}\right)}^{y} f^{\prime}\right] \\
& J_{3}=2 D^{0} D^{x}\left[\mu L_{\left(D^{y}, F, F^{\prime \prime}\right)}^{y} f+L_{\left(D^{y}, F, F\right)}^{y} f^{\prime \prime}\right] \\
& J_{x}=2 N_{x x}^{0} N_{x x}^{x}\left[L_{\left(N_{x x}^{y}, F, F\right)}^{y} f^{\prime}+L_{\left(N_{x x}^{y}, F, R\right)}^{y} r^{\prime}\right] \\
& J_{y}=2 N_{y y}^{0} N_{y y}^{x}\left[L_{\left(N_{y y}^{y}, F^{\prime}, F^{\prime}\right)}^{y} f+L_{\left(N_{y y}^{y}, F^{\prime}, R^{\prime}\right)}^{y}\right] \\
& J_{x y 1}=2 N_{x y}^{0} N_{x y}^{x}\left[L_{\left(N_{x y}^{y}, F^{\prime}, F\right)}^{y} f^{\prime}+L_{\left(N_{x y}^{y}, F^{\prime}, R\right)}^{y} r^{\prime}\right] \\
& J_{x y 2}=2 N_{x y}^{0} N_{x y}^{x}\left[L_{\left(N_{x y}^{y}, F, F^{\prime}\right)}^{y} f+L_{\left(N_{x y}^{y}, F, R^{\prime}\right)}^{y}\right] \\
& k_{1}=Q^{0} Q^{x}\left[L_{\left(Q^{y}, R, R\right)}^{y} r^{\prime}\right] \\
& k_{2}=Q^{0} Q^{x}\left[L_{\left(Q^{y}, R^{\prime}, R^{\prime}\right)}^{y} r\right] \\
& k_{x}=2 N_{x x}^{0} N_{x x}^{x}\left[L_{\left(N_{x x}^{y}, R, F\right)}^{y} f^{\prime}+L_{\left(N_{x x}^{y}, R, R\right)}^{y} r^{\prime}\right] \\
& k_{y}=2 N_{y y}^{0} N_{y y}^{x}\left[L_{\left(N_{y y}^{y}, R^{\prime}, F^{\prime}\right)}^{y} f+L_{\left(N_{y y}^{y}, R^{\prime}, R^{\prime}\right)}^{y} r\right] \\
& k_{x y 1}=2 N_{x y}^{0} N_{x y}^{x}\left[L_{\left(N_{x y}^{y}, R^{\prime}, F\right)}^{y} f^{\prime}+L_{\left(N_{x y}^{y}, R^{\prime}, R\right)}^{y} r^{\prime}\right] \\
& k_{x y} N_{x y}^{x}\left[L_{\left(N_{x y}^{y}, R, F^{\prime}\right)}^{y} f+L_{\left(N_{x y}^{y}, R, R^{\prime}\right)}^{y} r\right]
\end{aligned}
$$

where $Q^{0}$ is constant, $Q^{x}$ is a function of $x$ and $Q^{y}$ is a function of $y$ that their multiplication produces the following integral, given that the thickness $h$ is changing through the $x$ and $y$ directions.

$$
Q^{0} Q^{x} Q^{y}=Q_{(x, y)}=\int_{-h / 2}^{h / 2} \frac{E_{(z)} \eta_{(z)} \eta_{(z)}}{1+\mu} d z
$$

The second set of governing equations and boundary conditions can be derived similarly. For the sake of brevity, and due to the similarity with the ones above, those resulting formulations are omitted. 


\section{The derivation based on the RPT}

The sole aim of this section is to illustrate that for the FGM plates, using the refined plate theories (RPT) provides stability equations having coupled lateral and in-plane displacements variations, even if the physical neutral plane is considered as the reference plane. In RPT, the lateral displacement $w$ is decomposed into into $w_{b}$ and $w_{s}$. RPT treats the shear correction from down the level of the constitutive equations.

The shear component of the lateral displacement imposed with a selected function $\xi_{(z)}$ contributes to the in-plane displacements. $\xi_{(z)}$ gets transferred to the transverse shear strains and stresses through the kinematic relations. There are numerous versions of the traditional refined plate theory, e.g., [24, 2, 32, 6]; they normally differ in the adopted shear distribution function, $\xi_{(z)}$. Note that RPT herein refers to the form of the theory $\xi_{(z)}$ is general function. The general form of the constitutive equations of RPT is given as follows.

$$
\begin{aligned}
& \bar{u}_{(x, y, z)}=u_{(x, y)}-\left(z-z_{0}\right) \frac{\partial w_{b(x, y)}}{\partial x}-\xi_{(z)} \frac{\partial w_{s(x, y)}}{\partial x} \\
& \bar{v}_{(x, y, z)}=v_{(x, y)}-\left(z-z_{0}\right) \frac{\partial w_{b(x, y)}}{\partial y}-\xi_{(z)} \frac{\partial w_{s(x, y)}}{\partial y} \\
& \bar{w}_{(x, y, z)}=w_{s(x, y)}+w_{b(x, y)}
\end{aligned}
$$

In the RPT there is no need for the correction factor $K_{s}$, so the transverse shear stresses and strains are given as follows.

$$
\tau_{x z}=\tau_{z x}=\frac{E_{(z)}}{2(1+\mu)} \gamma_{x z} \quad \tau_{y z}=\tau_{z y}=\frac{E_{(z)}}{2(1+\mu)} \gamma_{y z}
$$

Starting from these constitutive equations and transverse shear strains and by implementing the same steps in Section $\square$ of finding the total potential energy $\Gamma$, then its second variation $\delta^{2} \Gamma$, then imposing the pre-buckling equilibrium state of zero lateral deflection $\left(w_{b}=w_{s}=\right.$ $0)$, provide $\delta_{e}^{2} \Gamma$ as follows.

$$
\delta_{e}^{2} \Gamma=\int_{-b}^{b} \int_{-a}^{a}\left(\Gamma_{e}\right) d x d y
$$

where $\Gamma_{e}=$

$$
\begin{aligned}
& =A\left(\begin{array}{l}
\delta u_{, y}^{2}+\delta v_{, y}^{2}+2 \mu \delta u_{, x} \delta v_{, y}+\left(\mu u_{, x}+v_{, y}\right)\left(\delta w_{b, y}+\delta w_{s, y}\right)^{2} \\
+\left(u_{, x}+\mu v_{, y}\right)\left(\delta w_{b, x}+\delta w_{s, x}\right)^{2}+\frac{1}{2}(1-\mu)\left(\left(\delta u_{, y}+\delta v_{, x}\right)^{2}+\delta w_{s, y}{ }^{2}+\delta w_{s, x}{ }^{2}\right) \\
+(1-\mu)\left(u_{, y}+v_{, x}\right)\left(\delta w_{b, x}+\delta w_{s, x}\right)\left(\delta w_{b, y}+\delta w_{s, y}\right)
\end{array}\right) \\
& -2 B\left(\delta w_{b, y y}\left(\mu \delta u_{, x}+\delta v_{, y}\right)+\delta w_{b, x x}\left(\delta u_{, x}+\mu \delta v_{, y}\right)+(1-\mu) \delta w_{b, x y}\left(\delta u_{, y}+\delta v_{, x}\right) \quad\right) \\
& +D\left(\delta w_{b, y y}^{2}+\delta w_{b, x x}{ }^{2}+2 \mu \delta w_{b, x x} \delta w_{b, y y}+2(1-\mu) \delta w_{b, x y}{ }^{2}\right) \\
& +Q_{1}\left(\delta w_{s, y y}^{2}+\delta w_{s, x x}^{2}+2 \mu \delta w_{s, x x} \delta w_{s, y y}+2(1-\mu) \delta w_{s, x y}^{2}\right) \\
& +2 Q_{2}\left(2(1-\mu) \delta w_{b, x y} \delta w_{s, x y}+\delta w_{b, x x}\left(\mu \delta w_{s, y y}+\delta w_{s, x x}\right)+\delta w_{b, y y}\left(\mu \delta w_{s, x x}+\delta w_{s, y y}\right)\right) \\
& +Q_{3}\left(\delta w_{s, y}^{2}+\delta w_{s, x}^{2}\right) \\
& -2 Q_{4}\left(\delta w_{s, y y}\left(\mu \delta u_{, x}+\delta v_{, y}\right)+\delta w_{s, x x}\left(\delta u_{, x}+\mu \delta v_{, y}\right)+(1-\mu) \delta w_{s, x y}\left(\delta u_{, y}+\delta v_{, x}\right) \quad\right)
\end{aligned}
$$


where the integrals $D, A$, and $B$ are the same ones given in Eq. 102, while the integrals $Q_{1} \ldots Q_{5}$ are given as follows.

$$
\begin{aligned}
Q_{1} & =\int_{-h / 2}^{h / 2} \frac{E_{(z)} \xi_{(z)} \xi_{(z)}}{1-\mu^{2}} d z & Q_{2} & =\int_{-h / 2}^{h / 2} \frac{\left(z-z_{0}\right) E_{(z)} \xi_{(z)}}{1-\mu^{2}} d z \\
Q_{3} & =\int_{-h / 2}^{h / 2} \frac{E_{(z)} \xi_{(z)}^{\prime}\left(2 \xi_{(z)}^{\prime}-1\right)}{1+\mu} d z & Q_{4} & =\int_{-h / 2}^{h / 2} \frac{E_{(z)} \xi_{(z)}}{1-\mu^{2}} d z
\end{aligned}
$$

Equation 41 shows that the variation of the bending component of the lateral displacement $\left(\delta w_{b}\right)$ is coupled with the variations of the in-plane displacements $(\delta u, \delta v)$ by the terms multiplied by the integral $B$. In addition, the variation of the shear component of the lateral displacement $\left(\delta w_{s}\right)$ is coupled with the variations of the in-plane displacements $(\delta u, \delta v)$ by the terms multiplied by the integral $Q_{4}$. Equating $B$ to zero makes the reference plane to be at the physical neutral plane where no coupling between the bending and the in-plane displacements. However, in order to obtain a set of equations with uncoupled lateral and in-plane displacements, $Q_{4}$ has also to be zero. Equating $Q_{4}$ to zero necessitates at least one of the following two statements. The first, $\xi_{(z)}$ is zero. The second, $E_{(z)}$ is constant and the integration of $\xi_{(z)}$ along the thickness is zero. if $\xi_{(z)}$ is zero, the RPT reduces to the classical plate theory (CPT). Otherwise, if the $E_{(z)}$ is constant, then the material is homogeneous. Therefore, for buckling analysis of FGM plates, using the RPT will always involve the solution for all the 4 variables $\left(\delta w_{b}, \delta w_{s}, \delta u, \delta v\right)$, even if the physical neutral plane is selected as the reference plane. In the case of homogeneous plates, the RPT provides much simpler buckling equations consisting only of the lateral displacement components $\left(\delta w_{b}, \delta w_{s}\right)$.

\section{Notes on the resulting formulations}

In the buckling equations based on FSDT, the lateral and in-plane displacements/rotations are found uncoupled at the physical neutral plane. This is also found true for the case of the refined-FSDT. In the case of the RPT, the lateral and in-plane displacements are found coupled, even at the physical neutral plane. So, the buckling equations of FGM plates based on the RPT contain all the four variables $\left(\delta w_{b}, \delta w_{s}, \delta u, \delta v\right)$ coupled together, while only the lateral displacements/rotations are contained in the stability equations of the FSDT and the refined-FSDT. The buckling equations based on the refined-FSDT, Eq. B6, consist of only two variables $\left(\delta w_{b}, \delta w_{s}\right)$, while those based on the FSDT, Eq. (24 and BO) ), consist of three variables $(\delta w, \delta \theta, \delta \varphi)$.

Although the number of variables is less in the refined-FSDT stability equations, they are not necessarily simpler than those of the FSDT. That because the former contain forthorder terms, while the latter contain terms with order no higher than two. Actually, the two sets of governing equations are similar in terms of simplicity, since the equivalent first-order system of each has six equations. Another point worth noticing is that the two variables of the stability equations of the refined-FSDT are coupled only at the resultant forces terms. The traditional refined theories come last in this comparison of simplicity.

The main advantage of refined-FSDT over FSDT is the elimination of the need for a shear correction factor $K_{s}$. Instead, it requires a shear distribution function $\eta_{(z)}$, which has to be selected wisely to satisfy the traction conditions at the top and bottom surfaces of the plate, and dictate the desired distribution of the transverse shear strains through the thickness. In the other hand, FSDT requires a shear correction factor $K_{s}$. For homogeneous isotropic plates, the often accepted shear correction factors are $(5 / 6)$ and $\left(\pi^{2} / 12\right)$. In such 
a case, FSDT formulations provide identical results to those of higher-order theories, even for thicker plates. In the case of the FGM plates, these correction factors are not necessarily accurate. Anyway, using those correction factors produces marginal error, unless for the case of thicker FGM plates with side-to-thickness ration $a / h$ less than 10 . However, many models are proposed to obtain modified correction factors for FGM, e.g. [3, 20, 19, 23]. In short, the EKM buckling formulations based on the FSDT and the refined-FSDT both are simple and practical to use.

\section{Conclusion}

The buckling equations and the boundary conditions of rectangular plates are derived in the frame of the EKM. The formulations are based on the FSDT, the refined-FSDT, and the RPT. The first presented derivation, that is based on the FSDT, is claimed to be the correct one versus the wrong one found in the literature. The second derivation, that is based on the refined-FSDT, is the first implementation of EKM to a refined plate theory. It is also the first time to implement the refined-FSDT in buckling analysis. The derivation based on the RPT is also discussed. It was concluded that the EKM buckling formulations based on the FSDT and refined-FSDT are equally simple in terms of equivalent first-order number of equations. The buckling formulations of FGM plates based on the RPT are found coupling the lateral and in-plane displacements even at the neutral plane.

The von Kármán geometrical nonlinearity is considered. General resultant in-plane forces, thickness variation, and material properties variation through the thickness are considered. The physical neutral plane is taken as the reference plane. The stability equations are derived according to the Trefftz criterion, i.e., the principle of minimum potential energy difference, using the variational calculus. EKM converts the buckling equations and boundary conditions from being 2D (PDE) into two sets of 1D ordinary differential equations (ODE), which are much easier to solve. Additional aspects of the plate problem, like the skew angle, and the effect of the elastic foundation, can easily be added to the derivation. The derivation is provided in detail so fellow researchers can easily redo the derivation for other configurations of their concern. By following the same exact steps illustrated here, the stability equations and boundary conditions of the plate based on other plate theories can also be obtained.

In future works, one can compare in detail the presented formulations in terms of accuracy, speed, and convergence. In addition, the effect of the shear correction function $\xi_{(z)}$ on the accuracy of the refined-FSDT should also be investigated.

\section{Conflict of interest}

The authors declare that they have no conflict of interest.

\section{References}

1. Chen, P.C.: Buckling analysis of a rectangular plate by the kantorovich method. International Journal of Mechanical Sciences 14(1), 15-24 (1972). URL https://doi.org/10.1016/0020-7403(72) 90003-3

2. Do, V.T., Pham, V.V., Nguyen, H.N.: On the development of refined plate theory for static bending behavior of functionally graded plates. Mathematical Problems in Engineering 2020 (2020). URL https://doi.org/10.1155/2020/2836763 
3. Efraim, E., Eisenberger, M.: Exact vibration analysis of variable thickness thick annular isotropic and fgm plates. Journal of Sound and Vibration 299(4), 720 - 738 (2007). URL https://doi.org/10. $1016 / j \cdot j$ sv. 2006.06 .068

4. Eisenberger, M., Alexandrov, A.: Buckling loads of variable thickness thin isotropic plates. Thin-Walled Structures 41(9), 871-889 (2003). URL https://doi.org/10.1016/S0263-8231(03)00027-2

5. Eslami, M.R.: Buckling and Postbuckling of Beams, Plates, and Shells. Springer (2018)

6. Farahmand, H.: Analytical solutions of bending and free vibration of moderately thick micro-plate via two-variable strain gradient theory. Journal of the Brazilian Society of Mechanical Sciences and Engineering 42, 1-11 (2020). URL https://doi.org/10.1007/s40430-020-02341-2

7. Grimm, T.R., Gerdeen, J.C.: Instability analysis of thin rectangular plates using the kantorovich method. Journal of Applied Mechanics 42(1), 110-114 (1975). URL https ://doi.org/10.1115/1.3423499

8. Hassan, A.H.A., Kurgan, N.: Buckling of thin skew isotropic plate resting on pasternak elastic foundation using extended kantorovich method. Heliyon 6(6), e04236 (2020). URL https://doi.org/10.1016/ J.heliyon.2020.e04236

9. Hassan, A.H.A., Kurgan, N.: The relations between the various critical temperatures of thin fgm plates. Journal of Applied and Computational Mechanics 6(Special Issue), 1404-1419 (2020). URL nttps: //doi.org/10.22055/jacm.2020.34697.2459

10. Jones, R.M.: Buckling of bars, plates, and shells. Bull Ridge Corporation (2006)

11. Kantorovich, L., Krylov, V.: Approximate Methods of Higher Analysis. Interscience, New York (1958)

12. Kerr, A.D.: An extension of the kantorovich method. Quarterly of Applied Mathematics 26(2), 219-229 (1968)

13. Kerr, A.D.: An extended kantorovich method for the solution of eigenvalue problems. International Journal of solids and structures 5(6), 559-572 (1969). URL https ://doi.org/10.1016/0020-7683(69) प्र0028-6

14. Kerr, A.D., Alexander, H.: An application of the extended kantorovich method to the stress analysis of a clamped rectangular plate. Acta Mechanica 6(2-3), 180-196 (1968). URL https://doi.org/10. $1007 / \mathrm{BF} 01170382$

15. Lopatin, A., Morozov, E.: Buckling of the sscf rectangular orthotropic plate subjected to linearly varying in-plane loading. Composite Structures 93(7), 1900-1909 (2011). URL https://doi.org/10.1016/ j.compstruct.2011.01.024

16. Lopatin, A., Morozov, E.: Buckling of a uniformly compressed rectangular sscf composite sandwich plate. Composite Structures 105, 108-115 (2013). URL https://doi.org/10.1016/j . compstruct. 2013.04 .036

17. Lopatin, A., Morozov, E.: Approximate buckling analysis of the ccff orthotropic plates subjected to inplane bending. International Journal of Mechanical Sciences 85, 38-44 (2014). URL https://doi. org/10.1016/j.ijmecsci.2014.05.003

18. Nguyen, H.N., Hong, T.T., Vinh, P.V., Quang, N.D., Thom, D.V., et al.: A refined simple first-order shear deformation theory for static bending and free vibration analysis of advanced composite plates. Materials 12(15), 2385 (2019). URL https://doi.org/10.3390/ma12152385

19. Nguyen, T.K., Sab, K., Bonnet, G.: Shear correction factors for functionally graded plates. Mechanics of Advanced Materials and Structures 14(8), 567-575 (2007). URL https://doi.org/10.1080/ $153764907016725 / 5$

20. Nguyen, T.K., Sab, K., Bonnet, G.: First-order shear deformation plate models for functionally graded materials. Composite Structures 83(1), 25 - 36 (2008). URL https://doi.org/10.1016/j. compstruct.2001.03.004

21. Qi, Y., Knight Jr, N.F.: A refined first-order shear-deformation theory and its justification by plane strain bending problem of laminated plates. International Journal of Solids and Structures 33(1), 49-64 (1996). URL https://doi.org/10.1016/0020-7683(95)00010-8

22. Reddy, J.N.: Theory and analysis of elastic plates and shells. CRC press (2006)

23. Shariyat, M., Alipour, M.: A novel shear correction factor for stress and modal analyses of annular FGM plates with non-uniform inclined tractions and non-uniform elastic foundations. International Journal of Mechanical Sciences 87, 60 - 71 (2014). URL https://doi.org/10.1016/j.ijmecsci.2014.05. 0.32

24. Shimpi, R.P.: Refined plate theory and its variants. AIAA journal 40(1), 137-146 (2002). URL nttps: //doi.org/10.2514/2.1622

25. Shufrin, I., Eisenberger, M.: Stability and vibration of shear deformable platesfirst order and higher order analyses. International Journal of Solids and Structures 42(3), 1225-1251 (2005). URL nttps: //doi.org/10.1016/j.ijsolstr.2004.06.067

26. Shufrin, I., Eisenberger, M.: Stability of variable thickness shear deformable platesfirst order and high order analyses. Thin-Walled Structures 43(2), 189-207 (2005). URL https://doi.org/10.1016/j. tws.2004.01.013 
27. Shufrin, I., Eisenberger, M.: Buckling of plates with variable in-plane forces. In: Analysis and Design of Plated Structures, vol. 2, pp. 26-55. Woodhead Publishing (2006). DOI https://doi.org/10.1533/ 9781845690960.26

28. Shufrin, I., Eisenberger, M.: Shear buckling of thin plates with constant in-plane stresses. International Journal of Structural Stability and Dynamics 7(02), 179-192 (2007). URL 10.1142/ S() 2.1945.5407(0)2.2:3x

29. Shufrin, I., Rabinovitch, O., Eisenberger, M.: Buckling of laminated plates with general boundary conditions under combined compression, tension, and sheara semi-analytical solution. Thin-Walled Structures 46(7), 925-938 (2008). URL https://doi.org/10.1016/j.tws.2008.01.040. A special issue to mark the Retiral of Professor Jim Rhodes, Founding Editor

30. Shufrin, I., Rabinovitch, O., Eisenberger, M.: Buckling of symmetrically laminated rectangular plates with general boundary conditions a semi analytical approach. Composite Structures 82(4), 521-531 (2008). URL 10.1016/j.compstruct.2007.02.003

31. Singhatanadgid, P., Singhanart, T.: The kantorovich method applied to bending, buckling, vibration, and $3 \mathrm{~d}$ stress analyses of plates: A literature review. Mechanics of advanced materials and structures 26(2), 170-188 (2019). URL https://doi.org/10.1080/15376494.2017.1365984

32. Tran, H.Q., Tran, M.T., Nguyen-Tri, P., et al.: A new four-variable refined plate theory for static analysis of smart laminated functionally graded carbon nanotube reinforced composite plates. Mechanics of Materials 142, 103294 (2020). URL https://doi.org/10.1016/j.mechmat.2019.103294

33. Xie, W.C., Elishakoff, I.: Buckling mode localization in rib-stiffened plates with misplaced stiffeners kantorovich approach. Chaos, Solitons \& Fractals 11(10), 1559-1574 (2000). URL https://doi. org/10.1016/S0960-0779(99)00078-8

34. Yu, T., Zhang, L.: The elastic wrinkling of an annular plate under uniform tension on its inner edge. International Journal of Mechanical Sciences 28(11), 729-737 (1986). URL https://doi.org/10. 1016/0020-7403(86)90020-2

35. Yuan, S., Jin, Y.: Computation of elastic buckling loads of rectangular thin plates using the extended kantorovich method. Computers \& structures 66(6), 861-867 (1998). URL https://doi.org/10. 1016/S0045-7949(97)00111-9

36. Yuan, S., Zhang, Y.: Further extension of the extended kantorovich method. Computational methods in engineering advances and applications 2, 1240-1245 (1992)

37. Zhang, D.G.: Modeling and analysis of fgm rectangular plates based on physical neutral surface and high order shear deformation theory. International Journal of Mechanical Sciences 68, 92-104 (2013). URL 10.1016/j.ijmecsci.2013.01.002

38. Zhang, D.G., Zhou, Y.H.: A theoretical analysis of fgm thin plates based on physical neutral surface. Computational Materials Science 44(2), 716 - 720 (2008). URL 10.1016/j.commatsci.2008.05. 016

39. Zhang, L., Yu, T.: The plastic wrinkling of an annular plate under uniform tension on its inner edge. International Journal of Solids and Structures 24(5), 497-503 (1988). URL https://doi.org/10. 1016/0020-7683(88)90004-2 